\title{
Limited Plasmodium sporozoite gliding motility in the absence of TRAP family adhesins
}

\author{
Konrad Beyer ${ }^{1 \dagger}$, Simon Kracht ${ }^{1 \dagger}$, Jessica Kehrer ${ }^{1,2}$, Mirko Singer ${ }^{1,3}$, Dennis Klug ${ }^{1,4}$ and
}

Friedrich Frischknecht ${ }^{1,2^{*}}$ (1)

\begin{abstract}
Background: Plasmodium sporozoites are the highly motile forms of malaria-causing parasites that are transmitted by the mosquito to the vertebrate host. Sporozoites need to enter and cross several cellular and tissue barriers for which they employ a set of surface proteins. Three of these proteins are members of the thrombospondin related anonymous protein (TRAP) family. Here, potential additive, synergistic or antagonistic roles of these adhesion proteins were investigated.

Methods: Four transgenic Plasmodium berghei parasite lines that lacked two or all three of the TRAP family adhesins TRAP, TLP and TREP were generated using positive-negative selection. The parasite lines were investigated for their capacity to attach to and move on glass, their ability to egress from oocysts and their capacity to enter mosquito salivary glands. One strain was in addition interrogated for its capacity to infect mice.
\end{abstract}

Results: The major phenotype of the TRAP single gene deletion dominates additional gene deletion phenotypes. All parasite lines including the one lacking all three proteins were able to conduct some form of active, if unproductive movement.

Conclusions: The individual TRAP-family adhesins appear to play functionally distinct roles during motility and infection. Other proteins must contribute to substrate adhesion and gliding motility.

Keywords: Anopheles, Transmission, Migration, Malaria, Adhesion

\footnotetext{
*Correspondence: freddy.frischknecht@med.uni-heidelberg.de

${ }^{\dagger}$ Konrad Beyer and Simon Kracht contributed equally

${ }^{1}$ Integrative Parasitology, Center for Infectious Diseases, Heidelberg

University Medical School, Im Neuenheimer Feld 344, 69120 Heidelberg, Germany

Full list of author information is available at the end of the article
} original author(s) and the source, provide a link to the Creative Commons licence, and indicate if changes were made. The images or other third party material in this article are included in the article's Creative Commons licence, unless indicated otherwise in a credit line to the material. If material is not included in the article's Creative Commons licence and your intended use is not permitted by statutory regulation or exceeds the permitted use, you will need to obtain permission directly from the copyright holder. To view a copy of this licence, visit http://creativecommons.org/licenses/by/4.0/. The Creative Commons Public Domain Dedication waiver (http://creativeco mmons.org/publicdomain/zero/1.0/) applies to the data made available in this article, unless otherwise stated in a credit line to the data. 


\section{Graphical Abstract}

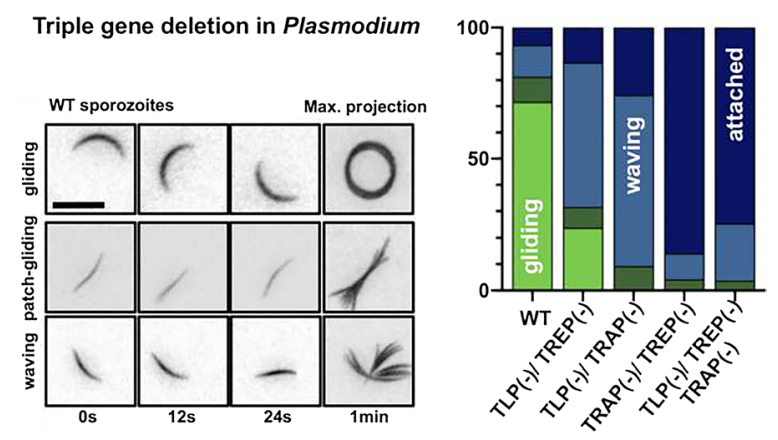

\section{Background}

Plasmodium sporozoites are the most versatile forms of the malaria parasite [1]. They develop within oocysts at the mosquito midgut and escape from these into the haemolymph of the mosquito [2,3]. Within the haemolymph, sporozoites are transported passively throughout the body cavity of the mosquito [4-6] and enter into the mosquito salivary glands. There sporozoites accumulate in large non-motile clusters while individual sporozoites progress slowly into the salivary duct [7-9]. During the bite of the mosquito 10-100 sporozoites are transmitted to the host [9-13]. Sporozoites, similar to most arthropod transmitted pathogens [14], are deposited in the dermis of the skin $[6,12,15-21]$. Within the dermis, sporozoites of avian parasites can already enter host cells and differentiate [22]. Sporozoites of mammalian infecting parasites have been shown to migrate at high speed exceeding $1 \mu \mathrm{m}$ per second, which strikingly is an order of magnitude faster than neutrophils [18, 23]. Sporozoites migrate through cells in the dermis, a capacity that is essential for entering the circulatory system [24, 25]. Sporozoites can enter both blood or lymph vessels [18] and those entering the blood are transported throughout the body to reach the liver $[26,27]$. In the liver sporozoites enter and differentiate within hepatocytes into red cell infecting merozoites $[25,28,29]$.

To achieve this remarkable journey, sporozoites employ a set of proteins that are specifically expressed only in sporozoites. The first protein that was shown to play a role in salivary gland invasion was the thrombospondinrelated anonymous protein (TRAP). A parasite line lacking the trap gene still formed sporozoites within oocysts but these were largely incapable of entering salivary glands [30, 31]. Haemolymph-derived sporozoites lacking trap were further incapable of performing productive motility [30] and showed a partial defect in substrate adhesion [32]. However, adhesive trap(-) sporozoites were still capable of performing a back-and-forth type of motility termed patch-gliding, which is typically found only in sporozoites isolated from the haemolymph [32]. Normal gliding motility of sporozoites, of other motile Plasmodium stages, and of related apicomplexan parasites is driven by an actin-myosin based motor located underneath the plasma membrane of the parasite and TRAP or TRAP-related proteins couple this motor to the extra-cellular substrate [33-36]. TRAP-related and TRAP family proteins share a similar domain structure spanning the plasma membrane [3]. They contain extracellular adhesive domains and they share a short cytoplasmic tail that is thought to couple the proteins to actin filaments [37]. Apart from TRAP, two more TRAP family members are expressed by sporozoites: the TRAP-like protein TLP and the TRAP-related extraordinary protein TREP, also named S6 or UOS3 [38-41] (Fig. 1A). Sporozoites lacking $t l p$ show only a slight phenotype in migration within the skin and hence some delayed infectivity in mice [38, 39, 42]. In contrast, sporozoites lacking trep have a strongly reduced capacity to enter salivary glands, but those that do enter the salivary gland subsequently infect mice normally [41]. Experiments employing optical traps to measure the forces that sporozoites can exert suggest that $\operatorname{trap}(-), \operatorname{trep}(-)$ and $t l p(-)$ sporozoites are all partially deficient in force generation [43, 44]. Intriguingly, the cohesive strengths of substrate adhesion sites are distinct in parasites lacking trap and trep [43].

Investigation of salivary gland sporozoites lacking $t l p$ indicates that TLP organizes macromolecular assemblies in order to couple the retrograde flow of actin filaments to force production $[44,45]$. TRAP is expressed early on in midgut derived sporozoites and still present and important for salivary gland sporozoites, while TREP expression wanes in the salivary glands although the protein is still present at this stage [40]. In contrast, TLP expression only starts after salivary gland entry [39] (Fig. 1A). Currently, it is not clear whether the individual TRAP family members exert their effects individually or 


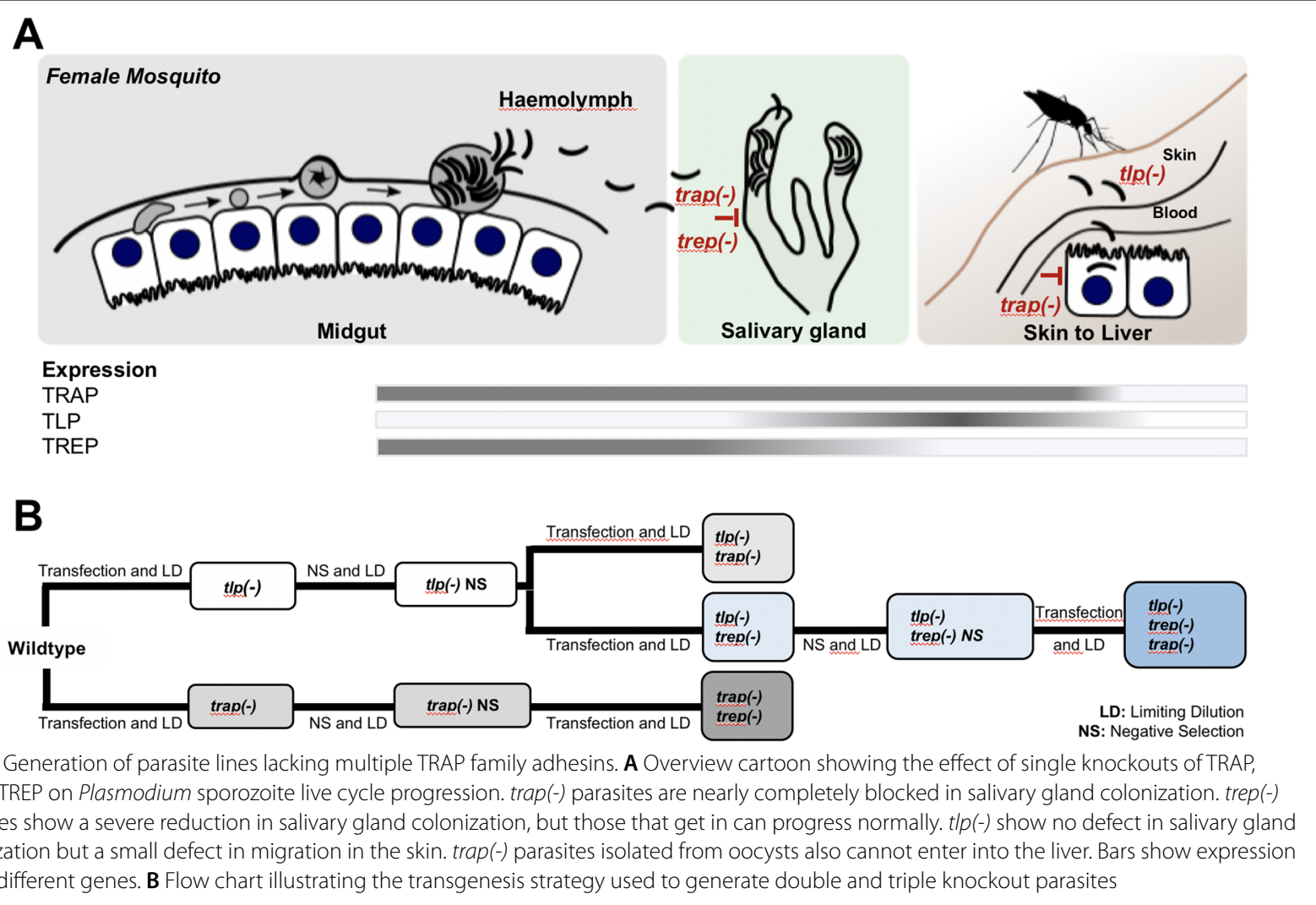

as an ensemble. Here, to address possible additive, synergistic, or antagonistic effects of the three proteins, and to investigate if sporozoite motility only depends on TRAP family adhesins, three parasite lines were generated and investigated that lacked two of the TRAP family members and one parasite line lacking all three.

\section{Methods}

\section{Handling of parasite lines}

Parasite lines were generated in blood stages and maintained in Swiss or NMRI mice through transfer of 10,000 blood stage parasites into the tail vein of naïve mice. Where possible (wild type and some mutants) parasites were cycled through mosquitoes prior to generating frozen aliquots. To this end, Anopheles stephensi mosquitoes were allowed to bite anesthetised mice, which contained gametocytes in their blood. Three weeks after infection two naive mice were bitten by the infected mosquitoes and blood stage parasitaemia was followed by daily blood smears. Whole blood was harvested by cardiac puncture. New infections were started by intraperitoneal injection of thawed aliquots.

\section{Generation of plasmids and parasite lines}

All generated plasmids were based on either the $\mathrm{Pb} 238$ or the $\mathrm{Pb} 301$ transfection vector $[46,47]$ containing the $5^{\prime}$ and $3^{\prime}$ UTRs of tlp or trep as well as the gene encoding the green fluorescent protein (GFP) [48]. All primer sequences are listed in Additional file 5: Table S1. Prior to transfection, all vectors were linearized with SacII and PmeI. Transfections were done as described in Janse et al. [49]. Schizonts were purified using a $55 \%$ nycodenz cushion followed by electroporation using the Amaxa T-cell Nucleofector kit. Parasites were maintained in 6-8 week old NMRI or CD1 mice provided with drinking water supplemented with pyrimethamine until a parasitaemia of more than $2 \%$ was reached.

$P b 262$ transfection vector $[3,50]$ : This vector uses the hDHFR-yFCU selection marker cassette [51] and the reporter gene mCherry under the control of the csp promoter. Using the $\mathrm{Pb} 238$ as parental vector, the selection marker cassette was replaced with the $d h f r 3^{\prime} \mathrm{UTR}-e f 1 \alpha$ $5^{\prime} \mathrm{UTR}-h D H F R-y F C U-d h f r 3^{\prime} \mathrm{UTR}$ from PlasmoGEM transfection vector [52], which was amplified with P600 and P601 and cloned with EcoRV HindIII into Pb238. Next, the $5^{\prime}$ UTR of csp was amplified from P. berghei gDNA with primers P207 and P208 and cloned into the vector with EcoRI and NdeI. The open reading frame of 
the red fluorescent protein encoding gene $m$ Cherry was amplified with primers P238 and P232 and inserted into the vector with NdeI/BamHI. The resulting vector is termed $\mathrm{Pb} 262$.

trap (-) and trap (-)NS: For the generation of trap(-) parasites the plasmid PbGEM-107890 was requested from PlasmoGEM [53, 54]. It replaces most of the TRAP coding sequence with the positive-negative selection marker $h D H F R-y F C U$. For negative selection [55] the drinking water of mice was supplemented with $1 \mathrm{mg} / \mathrm{ml} 5$-FC. The parasite line was already generated and characterized in a previous study [31] and used here as basis to generate the double mutant.

trap (-)/trep(-)/tlp(-)mCherry: The final TLP-KO transfection vector (PbCSmCherryYFCU_TLP-KO) was generated by amplifying the $5^{\prime}$ UTR of tlp from Plasmodium berghei gDNA with primers $\mathrm{P} 159$ and $\mathrm{P} 160$ and cloning into Pb238 via SacII/ NotI. The tlp 3' UTR was amplified with primers P161 and P162 and then cloned via HindIII/KpnI. Next, using HindIII and NotI restriction sites of the 'TLP plasmid' and Pb262, the selection cassette and the $m$ Cherry reporter gene of the $\mathrm{Pb} 262$ plasmid was inserted to create the final TLP-KO transfection plasmid as illustrated in Additional file 1: Fig. S1.

tlp(-)mCherryNS: For negative selection [55] the drinking water of mice infected with $t$ tp(-)mCherry parasites was supplemented with $1 \mathrm{mg} / \mathrm{ml} 5$-FC. Subsequently clonal parasites which lost the resistance cassette by homologous recombination were obtained by limiting dilution.

tlp(-)mCherry|trap(-): The PlasmoGem vector PbGEM107890 [53, 54] was transfected into selection marker free $t l p(-) m$ Cherry parasites. Clonal parasites were obtained by limiting dilution. Genotyping was performed as described in Additional file 1: Fig. S1.

tlp(-)mCherry|trep(-): In order to remove trep in the $t l p(-) m$ CherryNS parasites, the $5^{\prime}$ UTR of trep was amplified with primers P100 and P101, and cloned into Pb238 via SacII / NotI. The 3' UTR was amplified with primers P102 and P103 and then cloned via HindIII/KpnI. The resulting vector and $\mathrm{Pb} 262$ were digested with HindIII and NotI to insert the CSPmCHerryYFCU cassette, yielding PbCSmCherryYFCU_TREP-KO. This vector was used to create trap(-)|trep(-)mCherry (see below). For two of the planned knockout lines that already expressed mCherry in the $t l p$ locus $[\operatorname{TLP}(-) / \operatorname{TREP}(-)$ and TLP(-)/ TRAP(-)/TREP(-)], a TREP(-) transfection vector [PbYFCU_TREP(-)] was created that lacked the mCherry reporter gene. In order to remove the mCherry reporter gene and the CSP promoter, PbCSmChYFCU_TREP-KO was digested with NotI and EcoRV, blunted to get rid of any overhangs and finally re-ligated.
tlp(-)mCherry|trep(-)NS: The vector PbYFCU_TREP$\mathrm{KO}$ was transfected into selection marker free $t l p(-)$ $m$ Cherry parasites. Clonal parasites were obtained by limiting dilution. For negative selection [55] the drinking water of mice infected with $t$ lp(-)mCherry|trep(-) parasites was supplemented with $1 \mathrm{mg} / \mathrm{ml}$ 5-FC. Subsequently, clonal parasites which lost the resistance cassette by homologous recombination were obtained by limiting dilution. Genotyping was performed as described in Additional file 2: Fig. S2.

trap (-)|trep(-)mCherry: The PbCSmChYFCU_TREPKO transfection vector was transfected into trap $(-) N S$ parasites. Clonal parasites were obtained by limiting dilution. Genotyping was performed as described in Additional file 3: Fig. S3.

tlp(-)mCherry|trep(-)|trap(-): The PlasmoGem vector PbGEM-107890 [53, 54] was transfected into selection marker free $t l p(-) m C h e r r y \mid t r e p(-)$ parasites. Clonal parasites were obtained by limiting dilution. Genotyping was performed as described in Additional file 4: Fig. S4.

\section{Cryopreservation of parasites}

For longterm storage $100 \mu \mathrm{l}$ of blood containing parasites together with $200 \mu \mathrm{l}$ of freezing solution (10\% glycerol in Alsvers solution) was kept in liquid nitrogen.

\section{Isolation of parasites and gDNA preparation}

To isolate parasites for genotyping, at least $500 \mu \mathrm{l}$ of blood from an infected mouse was harvested via cardiac puncture. After the addition of $1 \mathrm{ml}$ PBS, red blood cells were lysed using $150 \mu \mathrm{l}$ of $1 \%$ saponin/ PBS. After centrifugation, the resulting parasite pellet was washed once with $1 \mathrm{ml}$ PBS and resuspended with $200 \mu \mathrm{l}$ PBS. Genomic DNA was isolated using a qiagen blood and tissue kit according to the manufacturers protocol.

\section{Mosquito infection}

Per infected mosquito cage, 20 million bloodstage parasites were transferred by intraperitoneal injection into 2 naïve NMRI or CD1 Swiss mice. After continued parasite growth for 3-5 days, the blood of the mice was monitored for the presence of gametocytes as estimated from observed exflagellation events. To do so, a drop of blood from the tail vein was placed on a glass slide and covered with a cover slip. After an incubation period of $10 \mathrm{~min}$ at room temperature, the slide was examined under a light microscope (Zeiss Axiostar, $100 \times$ objective; phase contrast). Three or more exflagellation events per field of view were deemed sufficient for subsequent mosquito infection.

To infect mosquitoes, mice were anesthetized with $100 \mu \mathrm{l}$ ketamine/xylazine $(100 \mathrm{mg} / \mathrm{ml}$ ketamin $+20 \mathrm{mg} /$ 
$\mathrm{ml}$ xylazine) and placed on top of a cage containing about $300 \mathrm{An}$. stephensi mosquitoes that were starved for at least $4 \mathrm{~h}$. Mosquitoes were allowed to bite infected mice for about $20 \mathrm{~min}$ and were then transferred into an incubator (T: $21^{\circ} \mathrm{C} ; 80 \%$ humidity) for parasite development.

\section{Mosquito dissection}

To obtain midguts, haemolymph, and salivary glands, infected mosquitoes were dissected under an SMZ 1500 binocular microscope (Nikon) using two syringes with needles. For the dissection of midguts, the last two abdominal segments were cut off and the remaining abdomen was detached from the thorax to expose the midgut, which was subsequently transferred into a reaction tube containing/filled with $200 \mu \mathrm{l}$ phosphate buffered saline (PBS). Salivary glands were obtained by carefully decapitating the mosquito with a pulling movement in a way that left the salivary glands attached to the head. Subsequently the salivary glands were separated from the head and transferred to a reaction tube supplied with $200 \mu \mathrm{l}$ PBS. To collect haemolymph the last two abdominal segments of the mosquito were cut off and the thorax pierced with a finely drawn pasteur pipette filled with PBS. The mosquito was gently flushed with PBS and the drops forming at the abdominal opening were collected on a piece of Parafilm. Drops were then transferred into an empty reaction tube using a pipette.

\section{Staining and counting of oocysts}

Oocyst numbers were determined between day 12 and 17 post infection. Midguts were isolated and incubated in $100 \mu \mathrm{l}$ PBS supplemented with 1\% NP40 for $20 \mathrm{~min}$ at room temperature. Staining of oocysts was performed using $100 \mu \mathrm{l}$ PBS supplemented with 0.1\% Mercurochrome. Midguts were incubated for up to $2 \mathrm{~h}$. After staining, the midguts were washed three times with PBS, transferred to a glass slide with a pasteur pipette and covered with a cover glass. Oocysts were counted manually with an Axiovert $200 \mathrm{M}$ epifluorescence microscope (Carl Zeiss) using transmission light, a green filter and an $10 \times$ air objective.

\section{Counting of sporozoites}

Midgut sporozoites were counted on days 14 and 16 after infection, haemolymph sporozoites on day 14-16 and salivary gland sporozoites on day 17 . To determine sporozoite numbers, salivary glands and midguts were collected from at least 10 mosquitos and smashed with a plastic pestle. All samples were counted under a light microscope with a $40 \times$ air objective using a haemocytometer (Neubauer improved) and phase contrast.

\section{Sporozoite gliding assays}

Haemolymph sporozoite gliding assays were performed on day 14 post infection and salivary gland sporozoite gliding assays on day 17 post infection. Haemolymph and salivary glands were collected as described above. To free sporozoites from salivary glands, glands were smashed using a plastic pestle before gliding assays were performed. Both haemolymph and salivary gland samples were centrifuged, the supernatant was discarded and sporozoite pellets were resuspended in $100 \mu \mathrm{l} 3 \% \mathrm{BSA} /$ RPMI solution to activate gliding motility. Resuspended sporozoites were transfered to a glass bottom 96-well plate and centrifuged at $1000 \mathrm{rpm}$ for $3 \mathrm{~min}$ at room temperature. Imaging was done with an Axiovert $200 \mathrm{M}$ epifluorescence microscope using a $25 \times$ glycerin objective. A series of timelapse videos with one frame per $3 \mathrm{~s}$ was obtained using a Prime BSI camera (Photometrics) mounted on the sideport of the microscope. Videos were analysed with Fiji (ImageJ). Sporozoites were classified in one of five gliding patterns (gliding, patch gliding, waving, attached, and floating) and sporozoite speed was determined using the ImageJ plugin 'Manual Tracking' [56].

\section{Transmission experiments}

To check the infectivity of the generated $\mathrm{KO}$ parasites, naive C57BL/6 mice were either exposed to infected mosquitoes or injected intravenously (i.v.) with salivary gland or haemolymph sporozoites. For infection of mice by bite, naive C57BL/ 6 mice were anesthetized with $100 \mu \mathrm{l} \mathrm{ketamin} /$ xylazin and then placed on top of netcovered cups containing 10 mosquitoes each. Mice were left on cups for around $20 \mathrm{~min}$. Blood-filled mosquitoes were subsequently dissected and salivary glands were collected to determine sporozoite numbers per mosquito. For i.v. injection, haemolymph and salivary gland sporozoites were collected and counted as described above. 10,000 haemolymph or 100 salivary gland sporozoites were injected into the tail vein of naive C57BL/6 mice. For both bite back and i.v. transmission experiments, parasitaemia was monitored through daily blood smears stained with Giemsa solution, starting on day 3 post infection. Mice were sacrificed on day 10 after infection at the latest to minimize suffering due to cerebral malaria and anaemia.

\section{Ethics statement}

All animal experiments were performed according to FELASA guidelines and were officially sanctioned by the responsible German authorities (Regierungspraesidium Karlsruhe) in accordance with the German Animal 
Welfare Act (Tierschutzgesetz). Female NMRI or CD1 swiss mice, ordered from JANVIER or Charles River Laboratories, were used for rearing of the parasites and infection of mosquitoes. Transmission experiments were performed using female C57BL/6 mice, ordered from Charles River Laboratories.

\section{Statistical analysis}

Statistical analyses were performed using GraphPad Prism 6.0 (GraphPad, San Diego, CA, USA). A value of $\mathrm{p}<0.05$ was considered significant. Specifically used tests are indicated in the figure legends.

\section{Results}

Generation of Plasmodium berghei parasite lines lacking two and three TRAP family adhesins

In order to generate parasite lines lacking more than one TRAP family gene, a strategy based on sequential positive and negative selection [51] was employed (Fig. 1B). Vectors for deletion of trep and tlp were engineered to replace the endogenous gene with the gene encoding for the red fluorescent protein mCherry controlled by the circumsporozoite protein $(c s p)$ promoter, which is strongly expressed throughout the life of sporozoites (Additional file 1: Fig. S1). The vector for the deletion of trap was obtained from PlasmoGEM [53, 54, 57], which also contained a cassette enabling positive-negative selection [31]. A clonal tlp knockout line was generated and the drug resistance cassette was recycled by negative selection [31]. Subsequently, vectors targeting the trap and trep genes were transfected into the $t l p(-)$ line (Additional file 1: Fig. S1, Additional file 2: Fig. S2). The trep $\mathrm{KO}$ vector was additionally transfected into trap(-) parasites that were generated previously yielding the line trep(-)/trap(-) [31] (Additional file 3: Fig. S3). A triple mutant line lacking all three TRAP family adhesins was obtained by negative selection of the trap $(-) / t l p(-)$ line followed by deletion of trep (Additional file 4: Fig. S4).

\section{Different developmental arrest of the parasite lines in mosquito midguts}

All parasite lines displayed normal growth of blood stages and infected mosquitoes at similar rates leading to comparable numbers of oocysts in mosquitoes (Fig. 2A, $B)$. Accordingly, the numbers of midgut sporozoites varied between cages infected with different mice but were all in the range of what were previously observed in our laboratory (compare range with e.g., [31]) (Fig. 2A). All parasite lines also showed normal to elevated numbers of sporozoites in the haemolymph (Fig. 2A) leading to a midgut-to-haemolymph sporozoite ratio that is comparable to wild type sporozoites (Fig. 2C). However, the numbers of sporozoites in the salivary glands as well as the midgut-to-salivary gland sporozoite ratio varied greatly (Fig. 2A, D). Sporozoite numbers of the trep(-)/ $t l p(-)$ line were in the range expected for trep $(-)$ parasites $[40,41]$, and all lines lacking trap showed very low numbers of salivary gland-resident sporozoites comparable to $\operatorname{trap}(-)$ sporozoites [30, 31].

\section{Sporozoites from all parasite lines could perform unproductive gliding}

Next sporozoites isolated from the mosquito haemolymph were analysed if they could attach to substrates, a prerequisite of gliding motility. This analysis revealed an attachment defect for all knockout lines in comparison to wild type controls (Fig. 3A). While around 35\% of control sporozoites attached to glass, only between 10 and $20 \%$ of the lines lacking two or three adhesins did so, suggesting that TRAP and TREP, the two TRAP-family adhesins expressed at this stage, contribute to the capacity of the sporozoite to adhere. Subsequently, motility and movement patterns of attached sporozoites were investigated. Three different types of sporozoite movement have been described [32, 58]: (i) productive gliding motility, during which the parasite progresses at high speed on a circular path that is dictated by the curvature of the sporozoite, (ii) patch gliding, during which the parasite moves over a single substrate adhesion point in a back-and-forth manner at peak speeds higher than those of productively gliding sporozoites and (iii) waving, during which sporozoites attach at one end to the substrate and move the parasite body around (Fig. 3A, B).

Quantification of the different movement patterns showed that more trep(-)/tlp(-) sporozoites were actively moving compared to the other double knockouts as well as the triple knockout (Fig. 3A). Also trep(-)/tlp() sporozoites were the only ones undergoing productive gliding motility, albeit at lower frequency and speed than wildtype parasites (Fig. 3A, C). In contrast, trap(-)/ $\operatorname{tlp}(-)$ and trap(-)/trep(-) sporozoites failed to undergo productive gliding. All parasite lines exhibited patchgliding behaviour at slightly different but consistently very low levels (Fig. 3A). Notably the two parasite lines lacking both TRAP and TREP showed much less waving compared to the lines where only one of these adhesion proteins is deleted. The fact that even the triple knockout shows some active motion suggests that other nonTRAP-family proteins are contributing to waving and patch-gliding motion, while TRAP is essential for sporozoites to perform active and directed motility.

Next, salivary-gland derived sporozoites were examined from the trep(-)/tlp(-) line, the only line that demonstrated some productive movement in haemolymph 


\begin{tabular}{llcc} 
A Parasite & MG Spz. & HL Spz. & SG Spz. \\
\hline WT & $48.000( \pm 15.000)$ & $3.000( \pm 700)$ & $11.000( \pm 6.500)$ \\
t/p(-)/trap(-) & $93.000( \pm 33.000)$ & $12.000( \pm 2.000)$ & $400( \pm 100)$ \\
t/p(-)/trep(-) & $62.000( \pm 45.000)$ & $11.000( \pm 7.000)$ & $900( \pm 500)$ \\
trap $(-) /$ trep $(-)$ & $41.000( \pm 20.000)$ & $4.000( \pm 600)$ & $100( \pm 100)$ \\
t|p(-)/trep(-)/trap$(-)$ & $61.000( \pm 54.000)$ & $11.000( \pm 9.000)$ & $150( \pm 150)$ \\
\hline
\end{tabular}
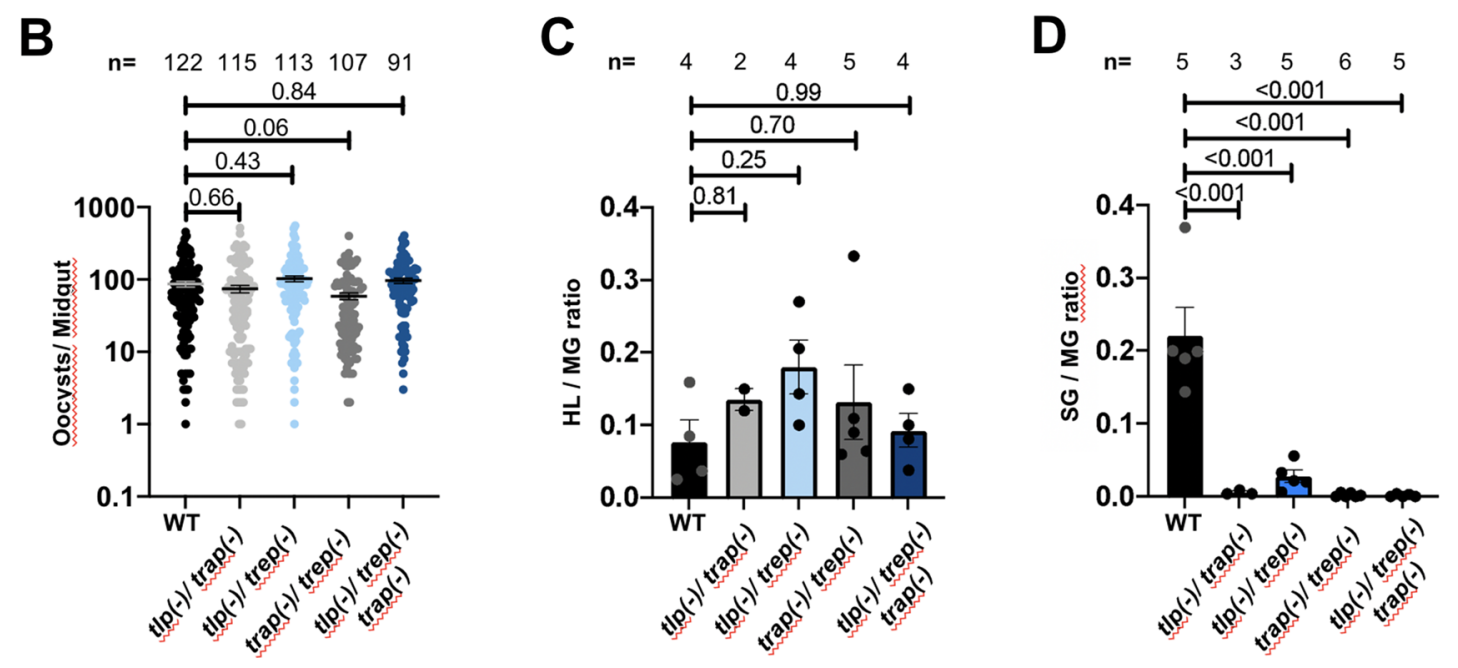

Fig. 2 Double and triple deletions can egress from oocysts but have defects in salivary gland entry. A Average numbers of midgut (MG), haemolymph ( $\mathrm{HL}$ ) and salivary gland (SG) derived sporozoites per mosquito \pm SD. Data from three independent feeding experiments. B Oocyst numbers of the different parasite lines. Black lines show mean \pm SEM. One-way Anova test with subsequent Dunnetts multiple comparison indicates significance ( $p$-value indicated above bars). Data from 2-4 independent experiments as follows: 2 for $t / p(-) / \operatorname{trap}(-), 3$ for $t / p(-) /$ trep $(-) /$ $\operatorname{trap}(-)$ and 4 for WT, t $/ p(-) / \operatorname{trep}(-)$ and trap(-)/trep(-). C Ratio of haemolymph to midgut sporozoite numbers for the different parasite lines. Bars show mean \pm SEM. One-way Anova test with subsequent Dunnetts multiple comparison indicates significance ( $p$-value indicated above bars). Data from 2 to 5 independent experiments as indicated above the bars. D Ratio of salivary gland to midgut sporozoite numbers for the different parasite lines. Bars show mean \pm SEM. One-way Anova test with subsequent Dunnetts multiple comparison indicates significance ( $p$-value indicated above bars)

sporozoites. Surprisingly, the rate of persistently gliding trep $(-) / t l p(-)$ salivary gland sporozoites was only slightly reduced compared to wildtype controls (Fig. 4A). However, similar to haemolymph trep(-)/tlp(-) sporozoites, salivary gland trep(-)/tlp(-) sporozoites moved significantly slower compared to wildtype (Fig. 4B). The observation that $\operatorname{trep}(-) / t l p(-)$ salivary gland sporozoites were capable to adhere suggests that TREP plays a role in adhesion of haemolymph sporozoites but possibly less so in salivary gland sporozoites.

\section{Transmission to mice is diminished in trep(-)/tlp(-) sporozoites}

The observation of productive movement in haemolymph and salivary gland sporozoites of the trep(-)/tlp(-) line suggested that these sporozoites might be able to infect mice. This possibility was tested in two ways: Either equal numbers of haemolymph or salivary gland derived sporozoites from the trep(-)/tlp(-) line or the wildtype control were injected intravenously into $\mathrm{C} 57 \mathrm{BL} / 6$, or mice were infected by allowing infected mosquitoes to feed (bite-back infection) (Fig. 5). Upon infection with haemolymph-derived trep(-)/tlp(-) sporozoites all seven infected mice developed blood-stage parasitaemia, yet with a delay of 1 day compared 


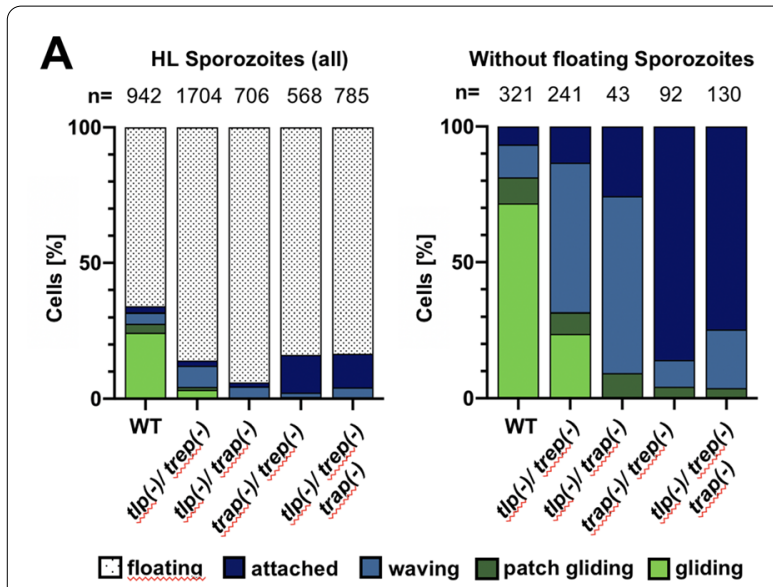

B

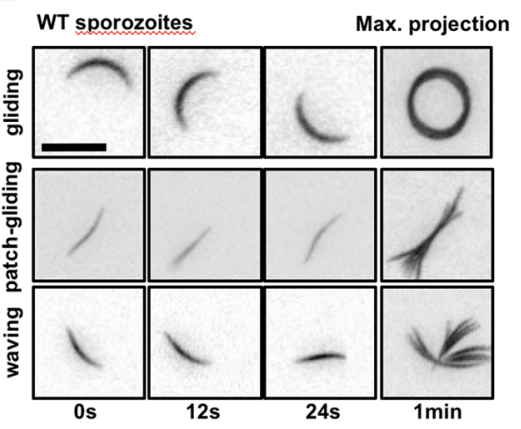

C

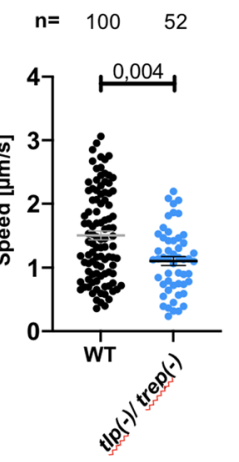

Fig. 3 Transgenic lines display weak adhesion and low rates of gliding motility. A Distribution of different movement patterns observed in haemolymph sporozoites. Data from three independent infections per line. B Example time lapse images of wild type sporozoites undergoing persistent gliding, patch-gliding and waving. Time is indicated below the images. Scale bar: $10 \mu \mathrm{m}$. $\mathbf{C}$ Speed of haemolymph derived sporozoites undergoing persistent gliding motility. Black lines show mean \pm SEM. Students t-test indicates significant differences. Data from two different infections per line

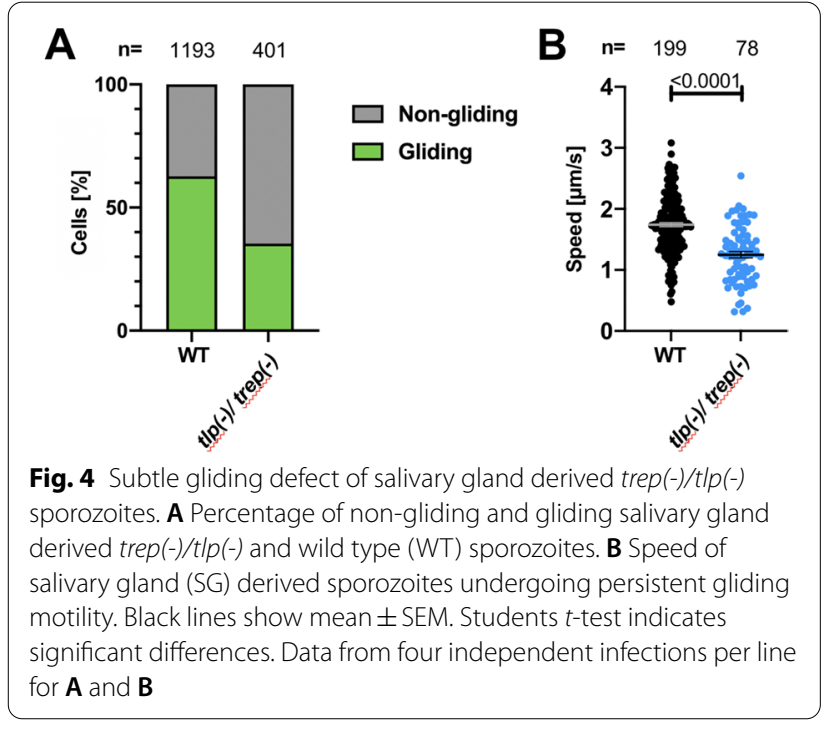

to wild type controls (Fig. 5A, B). This largely mimics the phenotype of the trep(-) parasite line [40]. In contrast, infection with just 100 salivary gland derived trep(-)/tlp(-) sporozoites showed no delay in blood stage onset or development compared to wildtype controls, although only $7 / 8$ mice injected with trep(-)/tlp(-) sporozoites became positive (Fig. 5A, C). Transmission by bite, however, showed a large decrease in transmission efficiency of trep(-)/tlp(-) sporozoites infected mosquitoes (Fig. 5A, D). Only 25\% (2/8) of mice became infected with trep(-)/tlp(-) and blood stage parasitaemia was delayed by 3 days compared to wildtype controls (Fig. 5A, D). This is likely a combined effect of the decreased number of salivary gland-resident sporozoites due to the TREP-mediated effect on salivary gland entry and the slightly diminished capacity of $t l p(-)$ sporozoites to migrate in the skin [40-42].

\section{Discussion}

The availability of a positive-negative selection system has allowed the sequential deletion of genes in P. berghei, as transgenic parasite lines can be selected for parasites that lost the resistance cassette. In theory, this approach allows for an unlimited number of sequential genetic modifications. To test this capacity, three members of a family of adhesins that are expressed on Plasmodium sporozoites were targeted to create double and triple knockout lines, evaluating potential synergistic, additive, or antagonistic roles of these proteins. The analysis of the generated double and triple deletion parasites showed that the defect in adhesion and gliding of the sporozoites largely mimicked the defect of the dominant gene, e.g., a parasite lacking trap showed the same defect in terms of gliding and salivary gland invasion whether just trap was deleted or whether in addition trep or tlp or both were deleted. The only observed difference was that parasite lines lacking both trap and trep had a smaller percentage of waving sporozoites compared to lines in which only one of those genes was absent (Fig. 3A). It is not clear what physiological role waving plays in sporozoite biology in vivo, but in vitro it indicates the capacity of the sporozoite to attach to a surface. Hence, this observation suggests that TREP and TRAP both play a role in forming single adhesions sites and complements previous work implicating TREP (S6) in the cohesive strength of 


\begin{tabular}{llccc}
\hline A & & & & \\
Parasite & Route of infection & Mice infected & Prepatency [davs] & Parasitemia dav 6 [\%] \\
\hline WT & by bite & $8 / 8$ & 3 & $1.7( \pm 0.7)$ \\
& $10 \mathrm{k} \mathrm{HL}$ iv & $7 / 7$ & 4 & $1.4( \pm 0.8)$ \\
& $100 \mathrm{SG}$ iv & $8 / 8$ & 4,5 & $0.8( \pm 0.4)$ \\
$t$ tho $-(-) /$ trep(-) & by bite & $2 / 8$ & 6 & $0,1( \pm 0.03)$ \\
& $10 \mathrm{k} \mathrm{HL}$ iv & $7 / 7$ & 5 & $0.4( \pm 0.3)$ \\
& $100 \mathrm{SG}$ iv & $7 / 8$ & 4,5 & $0.5( \pm 0.4)$ \\
\hline
\end{tabular}

B

B 10k Hemolymph sporozoites

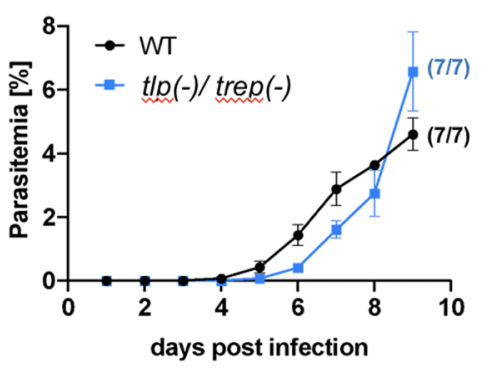

C

100 Salivary gland sporozoites

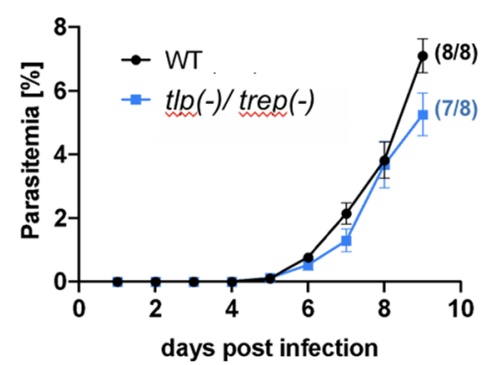

D Mosquito bite

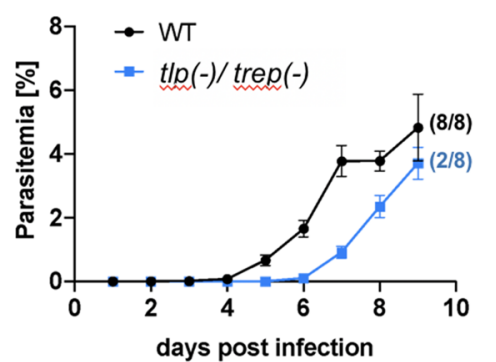

Fig. 5 trep(-)/tip(-) sporozoites transmit less efficiently to mice. A Summary of the transmission experiments with numbers of infected/total parasite injected (mosquito exposed) mice, prepatency and parasitaemia on day 6 given for the different parasite lines and the respective modes of transmission. Graphs display the development of blood stage parasitaemia in mice infected by intravenous injection of 10.000 haemolymph sporozoites. B or 100 salivary gland derived sporozoites. $\mathbf{C}$ and by the bites of 10 mosquitoes. D Wildtype control: black line, trep(-)/t/p(-): blue line; parasitaemia shows average for the two mice that became patent). Error bars indicate SEM

adhesion sites at the front of the parasite and TRAP at the rear end [43]. It is possible that TREP is secreted at the apex and allows sporozoites to form adhesions at the front-end enabling waving, which is followed by a second adhesion at the rear $[43,59]$. Consequently, a lack of TREP weakening this first adhesion could lower the efficiency of the formation of a secondary adhesion site, a prerequisite of gliding. The observation that TREP is only important for salivary gland entry suggests that TREP is important for sporozoites to attach to salivary glands. TRAP could help in generating or stabilizing these adhesion patches, while mainly functioning in motility.

Only the mutant lacking both trep and tlp could enter into salivary glands in sufficient numbers to allow further experiments. The efficiency of trep(-)/tlp(-) sporozoites to invade salivary glands was comparable to trep(-) parasites. The reduction of infectivity of trep(-)/tlp(-) sporozoites transmitted by mosquito bite is likely a result of the decreased numbers in salivary glands as for trep(-) parasites [60]. In addition there might be an effect of slower migration in the skin, but this would need further experiments using sensitive in vitro assays such as our recently established 3D gel-based assay [27] or in vivo imaging, which would require fluorescent parasite lines and much time. Both experiments are nearly impossible to achieve in parasite lines with strongly reduced salivary gland loads. Intriguingly, trep(-)/tlp(-) salivary gland sporozoites showed a similar infectivity of mice as $t l p(-)$ sporozoites when injected intravenously. This further suggests that a reduced number of transmitted sporozoites to the skin are the key reason for reduced by bite infection.

Taken together, no evidence was obtained of a synergistic or antagonistic effect of the investigated proteins. TRAP-family proteins appear to work independently of each other to enable sporozoite infection of salivary glands (TRAP, TREP) and efficient transmission to mice (TRAP, TLP). Interestingly, however, the observation that sporozoites lacking all three TRAP-family adhesins could still undergo some active motion, i.e., waving and patch gliding, suggests that additional proteins are important in sporozoite adhesion and the transition from adhesion to motility. It is important to note that sporozoites move in a stick-slip fashion that necessitates a continuous cycle of adhesion and deadhesion at distinct parts of the parasite [32]. Many other proteins are implicated in sporozoite gliding and among these are some that are likely to confer 
adhesive capacity to the sporozoite such as the complex PCRMP proteins [61], the circumsporozoite protein CSP and TRP1 [3]. These might contribute to modulating motility by influencing the adhesion-deadhesion cycle important for stick-slip locomotion. None of them were targeted here as deletion of $\operatorname{trp} 1$ inhibits sporozoite egress from oocysts [3] and deletion of csp inhibits sporozoite formation [62]. Furthermore, the MAEBL and AMA1 proteins, involved in sporozoite invasion of the salivary gland and liver, respectively [63-65], could play a role, as well as some recently identified rhoptry proteins that are involved in gliding motility $[66,67]$.

Lastly, there are also other proteins with no transmembrane domain that are linked to adhesion and gliding motility, such as LIMP and CelTOS [68, 69]. While nothing is known about their mechanistic function, some link to intracellular proteins could be expected and they might link to all or selected members of the TRAP family.

\section{Conclusions}

In conclusion, it was shown that the three TRAP-family members TRAP, TREP and TLP expressed in Plasmodium sporozoites can be deleted from the $P$. berghei genome with no effect on sporozoite formation and egress from oocysts. The observed phenotypes suggest that the three adhesins act independently from each other and that other proteins are also involved in sporozoite adhesion and motility. TRAP and TREP might be important for adhesion formation allowing sporozoite waving.

\section{Abbreviations}

CelTOS: Cell-traversal protein for ookinetes and sporozoites; CSP: Circumsporozoite protein; PCRMP: Plasmodium cysteine repeat modular proteins; CTRP: Circumsporozoite- and TRAP-related protein; S6: Sporozoite-specific protein 6; TLP: TRAP-like protein; TRAP: Thrombospondin related anonymous protein; TREP: TRAP-related protein; TRP1: Thrombospondin related protein 1; UOS3: Upregulated in oocyst sporozoites protein 3.

\section{Supplementary Information}

The online version contains supplementary material available at https://doi. org/10.1186/s12936-021-03960-3.

Additional file 1: Figure S1. Generation of $t / p(-) /$ trap (-) parasites via double homologous recombination. The cartoons (A, B) show the cloning strategy and primers used for genotyping with amplicon sizes of the resulting transgenic line indicated. Primer sequences are listed in table S2. The plasmid contains mCherry and the resistance marker yFCU for negative selection. (C) Resulting agarose gel picture after genotyping with the expected amplicon sizes given below. (D) Summary of primers and amplicon sizes for the genotyping PCR.

Additional file 2: Figure S2. Generation of t/p(-)/trep(-) parasites via double homologous recombination. The cartoon (A, B) shows the cloning strategy and primers used for genotyping with amplicon sizes of the resulting transgenic line indicated. Primer sequences are listed in table S2.
The plasmid contains mCherry and the resistance marker yFCU for negative selection. (C) Resulting Agarose gel picture after genotyping with the expected amplicon sizes given below. (D) Summary of primers and amplicon sizes for the genotyping PCR.

Additional file 3: Figure S3. Generation of trap(-)/trep(-) parasites via double homologous recombination. The cartoon (A, B) shows the cloning strategy and primers used for genotyping with amplicon sizes of the resulting transgenic line indicated. Primer sequences are listed in table S2. Plasmid contains mCherry and the resistance marker yFCU for negative selection. (C) Resulting agarose gel picture after genotyping with the expected amplicon sizes given below. (D) Summary of primers and amplicon sizes for the genotyping PCR.

Additional file 4: Figure S4. Generation of t/p(-)/trep(-)/trap(-) parasites via double homologous recombination. The cartoon $(\mathbf{A}, \mathbf{B}, \mathbf{C})$ shows the cloning strategy and primers used for genotyping with amplicon sizes of the resulting transgenic line indicated. Primer sequences are listed in table S2. The plasmid contains mCherry and the resistance marker yFCU for negative selection. (D) Resulting agarose gel picture after genotyping with the expected amplicon sizes given below. (E) Summary of primers and amplicon sizes for the genotyping PCR.

Additional file 5: Table S1. Primer list

\section{Acknowledgements}

We thank Miriam Reinig for mosquito breeding and Franziska Hentzschel for comments on the manuscript. A special thanks to the great people from PlasmoGEM for the TRAP KO vector and their very generous support over the years.

\section{Authors' contributions}

KB, SK, JK performed research, JK, MS, DK helped with research design, KB, SK, JK analysed data, FF supervised the study, acquired funding and wrote the manuscript. All authors commented on the manuscript. All authors read and approved the final manuscript.

\section{Funding}

Open Access funding enabled and organized by Projekt DEAL. This work was supported by a grant from the Human Frontier Science Program (RGY0071/2011), the Deutsche Forschungsgemeinschaft (DFG, German Research Foundation)_-Project Number 240245660_SFB 1129 and the European Research Council (ERC StG 281719). KB and DK were members of the HBIGS graduate school and FF was a member of CellNetworks cluster of excellence at Heidelberg University.

\section{Availability of data and materials}

All generated data are displayed within the manuscript and generated parasite lines are available from the corresponding author on request.

\section{Declarations}

Ethics approval and consent to participate

Animal work was approved by the concerned German authorities (Regierungspräsidum Karlsruhe).

\section{Consent for publication}

All authors have read the paper and consent to its publication. No patientderived data requiring consent is included in the manuscript.

\section{Competing interests}

The authors declare to have no competing interests.

\section{Author details}

${ }^{1}$ Integrative Parasitology, Center for Infectious Diseases, Heidelberg University Medical School, Im Neuenheimer Feld 344, 69120 Heidelberg, Germany. ${ }^{2}$ German Center for Infection Research, Partner Site Heidelberg, 69120 Heidelberg, Germany. ${ }^{3}$ Present Address: Experimental Parasitology, Faculty of Veterinary Medicine, Ludwig-Maximilians-University Munich, Lena-Christ-Straße 48, Planegg, 82152 Munich, Germany. ${ }^{4}$ Present Address: Université de Strasbourg, 
CNRS UPR9022, INSERM U963, Institut de Biologie Moléculaire et Cellulaire, 67000 Strasbourg, France.

Received: 27 May 2021 Accepted: 19 October 2021

Published online: 30 October 2021

\section{References}

1. Frischknecht F, Matuschewski K. Plasmodium sporozoite biology. Cold Spring Harb Perspect Med. 2017;7: a025478.

2. Orfano AS, Nacif-Pimenta R, Duarte APM, Villegas LM, Rodrigues NB, Pinto $L C$, et al. Species-specific escape of Plasmodium sporozoites from oocysts of avian, rodent, and human malarial parasites. Malar J. 2016;15:394.

3. Klug D, Frischknecht F. Motility precedes egress of malaria parasites from oocysts. Elife. 2017;6: e19157.

4. Akaki M, Dvorak JA. A chemotactic response facilitates mosquito salivary gland infection by malaria sporozoites. J Exp Biol. 2005;208:3211-8.

5. Frischknecht F, Martin B, Thiery I, Bourgouin C, Menard R. Using green fluorescent malaria parasites to screen for permissive vector mosquitoes. Malar J. 2006;5:23.

6. Douglas RG, Amino R, Sinnis P, Frischknecht F. Active migration and passive transport of malaria parasites. Trends Parasitol. 2015;31:357-62.

7. Sterling CR, Aikawa M, Vanderberg JP. The passage of Plasmodium berghei sporozoites through the salivary glands of Anopheles stephensi: an electron microscope study. J Parasitol. 1973;59:593-605.

8. Pimenta PF, Touray M, Miller L. The journey of malaria sporozoites in the mosquito salivary gland. J Eukaryot Microbiol. 1994;41:608-24.

9. Frischknecht F, Baldacci P, Martin B, Zimmer C, Thiberge S, Olivo-Marin $\mathrm{JC}$, et al. Imaging movement of malaria parasites during transmission by Anopheles mosquitoes. Cell Microbiol. 2004;6:687-94.

10. Beier JC, Davis JR, Vaughan JA, Noden BH, Beier MS. Quantitation of Plasmodium falciparum sporozoites transmitted in vitro by experimentally infected Anopheles gambiae and Anopheles stephensi. Am J Trop Med Hyg. 1991:44:564-70.

11. Beier JC, Onynago FK, Koros JK, Ramadhan M, Ogwang R, Wirtz RA, et al. Quantitation of malaria sporozoites transmitted in vitro during salivation by wild Afrotropical Anopheles. Med Vet Entomol. 1991;5:71-9.

12. Medica DL, Sinnis P. Quantitative dynamics of Plasmodium yoelii sporozoite transmission by infected anopheline mosquitoes. Infect Immun. 2005;73:4363-9.

13. Yamauchi LM, Coppi A, Snounou G, Sinnis P. Plasmodium sporozoites trickle out of the injection site. Cell Microbiol. 2007;9:1215-22.

14. Frischknecht $F$. The skin as interface in the transmission of arthropodborne pathogens. Cell Microbiol. 2007;9:1630-40.

15. Sidjanski SP, Vanderberg JP, Sinnis P. Anopheles stephensi salivary glands bear receptors for region I of the circumsporozoite protein of Plasmodium falciparum. Mol Biochem Parasitol. 1997;90:33-41.

16. Vanderberg JP, Frevert U. Intravital microscopy demonstrating antibodymediated immobilisation of Plasmodium berghei sporozoites injected into skin by mosquitoes. Int J Parasitol. 2004;34:991-6.

17. Amino R, Ménard R, Frischknecht F. In vivo imaging of malaria parasites - recent advances and future directions. Curr Opin Microbiol. 2005;8:407-14.

18. Amino R, Thiberge S, Martin B, Celli S, Shorte S, Frischknecht F, et al. Quantitative imaging of Plasmodium transmission from mosquito to mammal. Nat Med. 2006;12:220-4.

19. Ménard R, Tavares J, Cockburn I, Markus M, Zavala F, Amino R. Looking under the skin: the first steps in malarial infection and immunity. Nat Rev Microbiol. 2013;11:701-12.

20. Aliprandini E, Tavares J, Panatieri RH, Thiberge S, Yamamoto MM, Silvie O, et al. Cytotoxic anti-circumsporozoite antibodies target malaria sporozoites in the host skin. Nat Microbiol. 2018:3:1224-33.

21. Hopp CS, Kanatani S, Archer NK, Miller RJ, Liu H, Chiou KK, et al. Comparative intravital imaging of human and rodent malaria sporozoites reveals the skin is not a species-specific barrier. EMBO Mol Med. 2021;13: e11796.

22. Frevert U, Späth GF, Yee H. Exoerythrocytic development of Plasmodium gallinaceum in the White Leghorn chicken. Int J Parasitol. 2008;38:655-72.

23. Hopp CS, Chiou K, Ragheb DRT, Salman AM, Khan SM, Liu AJ, et al. Longitudinal analysis of Plasmodium sporozoite motility in the dermis reveals component of blood vessel recognition. Elife. 2015;4: e07789.
24. Amino R, Giovannini D, Thiberge S, Gueirard P, Boisson B, Dubremetz $\mathrm{JF}$, et al. Host cell traversal is important for progression of the malaria parasite through the dermis to the liver. Cell Host Microbe. 2008;3:88-96.

25. Loubens M, Vincensini L, Fernandes P, Briquet S, Marinach C, Silvie O. Plasmodium sporozoites on the move: switching from cell traversal to productive invasion of hepatocytes. Mol Microbiol. 2021;115:870-81.

26. Coppi A, Tewari R, Bishop JR, Bennett BL, Lawrence R, Esko JD, et al. Heparan sulfate proteoglycans provide a signal to Plasmodium sporozoites to stop migrating and productively invade host cells. Cell Host Microbe. 2007;2:316-27.

27. Ripp J, Kehrer J, Smyrnakou X, Tisch N, Tavares J, Amino R, et al. Malaria parasites differentially sense environmental elasticity during transmission. EMBO Mol Med. 2021;13: e13933.

28. Tavares J, Formaglio P, Thiberge S, Mordelet E, Van Rooijen N, Medvinsky $\mathrm{A}$, et al. Role of host cell traversal by the malaria sporozoite during liver infection. J Exp Med. 2013;210:905-15.

29. Prudêncio M, Rodriguez A, Mota MM. The silent path to thousands of merozoites: the Plasmodium liver stage. Nat Rev Microbiol. 2006:4:849-56.

30. Sultan AA, Thathy V, Frevert U, Robson KJH, Crisanti A, Nussenzweig V, et al. TRAP is necessary for gliding motility and infectivity of Plasmodium sporozoites. Cell. 1997;90:511-22.

31. Klug D, Goellner S, Kehrer J, Sattler J, Strauss L, Singer M, et al. Evolutionarily distant I domains can functionally replace the essential ligandbinding domain of Plasmodium trap. Elife. 2020;9: e57572.

32. Münter S, Sabass B, Selhuber-Unkel C, Kudryashev M, Hegge S, Engel U, et al. Plasmodium sporozoite motility is modulated by the Turnover of Discrete Adhesion Sites. Cell Host Microbe. 2009;6:551-62.

33. Meissner M, Ferguson DJP, Frischknecht F. Invasion factors of apicomplexan parasites: essential or redundant? Curr Opin Microbiol. 2013;16:438-44.

34. Montagna GN, Matuschewksi K, Buscaglia CA. Plasmodium sporozoite motility: an update. Front Biosci. 2012;17:726-44.

35. Heintzelman MB. Gliding motility in apicomplexan parasites. Semin Cell Dev Biol. 2015;46:135-42.

36. Tardieux I, Baum J. Reassessing the mechanics of parasite motility and host-cell invasion. J Cell Biol. 2016;214:507-15.

37. Kappe S, Bruderer T, Gantt S, Fujioka H, Nussenzweig V, Ménard R. Conservation of a gliding motility and cell invasion machinery in Apicomplexan parasites. J Cell Biol. 1999;147:937-43.

38. Heiss K, Nie H, Kumar S, Daly TM, Bergman LW, Matuschewski K. Functional characterization of a redundant Plasmodium TRAP family invasin, TRAP-like protein, by aldolase binding and a genetic complementation test. Eukaryot Cell. 2008;7:1062-70.

39. Moreira CK, Templeton TJ, Lavazec C, Hayward RE, Hobbs CV, Kroeze $\mathrm{H}$, et al. The Plasmodium TRAP/MIC2 family member, TRAP-like protein (TLP), is involved in tissue traversal by sporozoites. Cell Microbiol. 2008;10:1505-16.

40. Steinbuechel M, Matuschewski K. Role for the Plasmodium sporozoitespecific transmembrane protein $\mathrm{S} 6$ in parasite motility and efficient malaria transmission. Cell Microbiol. 2009;1 1:279-88.

41. Combe A, Moreira CK, Ackerman S, Thiberge S, Templeton TJ, Ménard R. TREP, a novel protein necessary for gliding motility of the malaria sporozoite. Int J Parasitol. 2009:39:489-96.

42. Hellmann JK, Münter S, Kudryashev M, Schulz S, Heiss K, Müller AK, et al. Environmental constraints guide migration of malaria parasites during transmission. PLoS Pathog. 2011;7: e1002080.

43. Hegge S, Uhrig K, Streichfuss M, Kynast-Wolf G, Matuschewski K, Spatz $J$, et al. Direct manipulation of malaria parasites with optical tweezers reveals distinct functions of Plasmodium surface proteins. ACS Nano. 2012;6:4648-62.

44. Quadt KA, Streichfuss M, Moreau CA, Spatz JP, Frischknecht F. Coupling of retrograde flow to force production during malaria parasite migration. ACS Nano. 2016;10:2091-102.

45. Hellmann JK, Perschmann N, Spatz JP, Frischknecht F. Tunable substrates unveil chemical complementation of a genetic cell migration defect. Adv Healthc Mater. 2013;2:1162-9.

46. Deligianni E, Morgan RN, Bertuccini L, Kooij TWA, Laforge A, Nahar C, et al. Critical role for a stage-specific actin in male exflagellation of the malaria parasite. Cell Microbiol. 2011;13:1714-30. 
47. Kooij TWA, Carlton JM, Bidwell SL, Hall N, Ramesar J, Janse CJ, et al. A Plasmodium whole-genome synteny map: Indels and synteny breakpoints as foci for species-specific genes. PLoS Pathog. 2005;1: e0010044.

48. Beyer K. Collective motion and adhesin dynamics of Plasmodium sporozoites. Thesis, University of Heidelberg. 2017. https://archiv.ub.uni-heide |berg.de/volltextserver/23561/1/Thesis_KonradBeyer.pdf.

49. Janse CJ, Ramesar J, Waters AP. High-efficiency transfection and drug selection of genetically transformed blood stages of the rodent malaria parasite Plasmodium berghei. Nat Protoc. 2006;1:346-56.

50. Singer M, Marshall J, Heiss K, Mair GR, Grimm D, Mueller AK, et al. Zinc finger nuclease-based double-strand breaks attenuate malaria parasites and reveal rare microhomology-mediated end joining. Genome Biol. 2015;16:249.

51. Braks JAM, Franke-Fayard B, Kroeze H, Janse CJ, Waters AP. Development and application of a positive-negative selectable marker system for use in reverse genetics in Plasmodium. Nucleic Acids Res. 2006;34: e39.

52. Pfander C, Anar B, Schwach F, Otto TD, Brochet M, Volkmann K, et al. A scalable pipeline for highly effective genetic modification of a malaria parasite. Nat Methods. 2011;8:1078-84.

53. Gomes AR, Bushell E, Schwach F, Girling G, Anar B, Quail MA, et al. A genome-scale vector resource enables high-throughput reverse genetic screening in a malaria parasite. Cell Host Microbe. 2015;17:404-13.

54. Schwach F, Bushell E, Gomes AR, Anar B, Girling G, Herd C, et al. PlasmoGEM, a database supporting a community resource for largescale experimental genetics in malaria parasites. Nucleic Acids Res. 2015;43:D1176-82.

55. Orr RY, Philip N, Waters AP. Improved negative selection protocol for Plasmodium berghei in the rodent malarial model. Malar J. 2012;11:103.

56. Schindelin J, Rueden CT, Hiner MC, Eliceiri KW. The ImageJ ecosystem: an open platform for biomedical image analysis. Mol Reprod Dev. 2015:82:518-29.

57. Bushell E, Gomes AR, Sanderson T, Anar B, Girling G, Herd C, et al. Functional profiling of a Plasmodium genome reveals an abundance of essential genes. Cell. 2017;170:260-72.e8.

58. Vanderberg JP. Studies on the motility of Plasmodium sporozoites. J Protozool. 1974;21:527-37.

59. Hegge S, Kudryashev M, Barniol L, Frischknecht F. Key factors regulating Plasmodium berghei sporozoite survival and transformation revealed by an automated visual assay. FASEB J. 2010;24:5003-12.

60. Aleshnick M, Ganusov VV, Nasir G, Yenokyan G, Sinnis P. Experimental determination of the force of malaria infection reveals a non-linear relationship to mosquito sporozoite loads. PLoS Pathog. 2020;16: e1008181.
61. Douradinha B, Augustijn KD, Moore SG, Ramesar J, Mota MM, Waters AP, et al. Plasmodium cysteine repeat modular proteins 3 and 4 are essential for malaria parasite transmission from the mosquito to the host. Malar J. 2011;10:71.

62. Ménard R, Sultan AA, Cortest C, Ahszulert R, van Dijk MR, Janse CJ, et al. Circumsporozoite protein is required for development of malaria sporozoites in mosquitoes. Nature. 1997;385:336-40.

63. Kariu T, Yuda M, Yano K, Chinzei Y. MAEBL is essential for malarial sporozoite infection of the mosquito salivary gland. J Exp Med. 2002;195:1317-23.

64. Saenz FE, Balu B, Smith J, Mendonca SR, Adams JH. The transmembrane isoform of Plasmodium falciparum MAEBL is essential for the invasion of Anopheles salivary glands. PLoS ONE. 2008;3: e0002287.

65. Yang ASP, Lopaticki S, O'Neill MT, Erickson SM, Douglas DN, Kneteman NM, et al. AMA1 and MAEBL are important for Plasmodium falciparum sporozoite infection of the liver. Cell Microbiol. 2017;19: e12745.

66. Bantuchai S, Nozaki M, Thongkukiatkul A, Lorsuwannarat N, Tachibana $\mathrm{M}$, Baba M, et al. Rhoptry neck protein 11 has crucial roles during malaria parasite sporozoite invasion of salivary glands and hepatocytes. Int J Parasitol. 2019;49:725-35.

67. Nozaki M, Baba M, Tachibana M, Tokunaga N, Torii M, Ishino T. Detection of the Rhoptry Neck Protein Complex in Plasmodium sporozoites and its contribution to sporozoite invasion of salivary glands. mSphere. 2020;5: e00325-20.

68. Santos JM, Egarter S, Zuzarte-Luís V, Kumar H, Moreau CA, Kehrer J, et al. Malaria parasite LIMP protein regulates sporozoite gliding motility and infectivity in mosquito and mammalian hosts. Elife. 2017;6: e24109.

69. Steel RWJ, Pei Y, Camargo N, Kaushansky A, Dankwa DA, Martinson T, et al. Plasmodium yoelii S4/CelTOS is important for sporozoite gliding motility and cell traversal. Cell Microbiol. 2018;20: e12817.

\section{Publisher's Note}

Springer Nature remains neutral with regard to jurisdictional claims in published maps and institutional affiliations.
Ready to submit your research? Choose BMC and benefit from:

- fast, convenient online submission

- thorough peer review by experienced researchers in your field

- rapid publication on acceptance

- support for research data, including large and complex data types

- gold Open Access which fosters wider collaboration and increased citations

- maximum visibility for your research: over 100M website views per year

At BMC, research is always in progress.

Learn more biomedcentral.com/submissions 